\title{
Transverse Field Effects of Al Concentration on Magnetic Properties of B2-FeAl Nanoparticle
}

\author{
H. YAŞAR $\mathrm{OCAK}^{a}$, G. Dikici YILdiz ${ }^{b}$, Y. GÖKTÜRK YILDIZ ${ }^{c, *}$, \\ B. SAATÇI ${ }^{d}$, R. BAŞAR ${ }^{a}$, G. SARIOĞLU ${ }^{e}$ \\ ${ }^{a}$ Department of Physics, Dumlupinar University, 43100, Kütahya, Turkey \\ ${ }^{b}$ Department of Physics, Kirıkkale University, 71450, Kirıkkale, Turkey \\ ${ }^{c}$ Department of Electronics and Automation, Kirıkkale University, 71450, Kirıkkale, Turkey \\ ${ }^{d}$ Department of Physics, Erciyes University, 38280, Kayseri, Turkey \\ ${ }^{e}$ Vocational School of Gediz, Dumlupınar University, 43100, Kütahya, Turkey
}

Received: 19.05.2020 \& Accepted: 28.09.2020

Doi: $10.12693 /$ APhysPolA.139.20

*e-mail: gokturk@kku.edu.tr

\begin{abstract}
Transverse field dependence of magnetic properties of the B2-FeAl nanoparticle (B2-FeAl-np) is investigated by using the effective field theory (or the Kaneyoshi theory) at $H=0$ and $H=0.25$. It is found that the magnetization and the Curie temperature of B2-FeAl-np decrease as the transverse field increases. A similar decrease in the magnetization and the Curie temperature of $B 2$-FeAl is experimentally observed by Plazaola et al. with the increase $(27.5 \%, 30 \%, 32.5 \%$ and $35 \%$ ) of the $\mathrm{Al}$ content in $B 2-\mathrm{FeAl}$. These theoretical and experimental results revealed that the increase of the $\mathrm{Al}$ content in B2-FeAl-np causes an increase in the transverse field of B2-FeAl-np. Thus, the magnetization and the Curie temperature of $B 2-\mathrm{FeAl}$-np decrease. However, the ferromagnetic and antiferromagnetic spin splitting of paramagnetic $\mathrm{Fe}$ and $\mathrm{Al}$ atoms is obtained by a non-zero external magnetic field $(H=0.25)$ at $T>T_{\mathrm{C}}$.
\end{abstract}

topics: $B 2-\mathrm{FeAl}$ nanoparticle, magnetism, transverse Ising model, effective field theory

\section{Introduction}

FeAl alloys have been extensively studied because of their particular mechanical, electrical, and magnetic properties. These alloys are potential candidates to replace stainless steels due to their high strength, excellent corrosion resistance and low density. Since the FeAl alloys show different magnetic and physical properties with their composition change and heat treatment, FeAl-based magnetic systems have been extensively investigated [1-3]. The studied issues included the magnetic properties of the binary $\mathrm{Fe}_{90} \mathrm{Al}_{10}, \mathrm{Fe}_{80} \mathrm{Al}_{20}$ and $\mathrm{Fe}_{50} \mathrm{Al}_{50}$ alloys [4], magnetic properties of the FeAl alloys [5], magnetic hysteresis properties of the Fe, FeAl alloys and $\mathrm{Fe} / \mathrm{Al}$ core/shell cluster composite [6], temperature dependence of magnetization and susceptibility and magnetic hysteresis loops of the B2-type $\mathrm{FeAl}$ alloys [7], magnetic hysteresis properties of the D03 and B2-types FeAl alloys [8], temperature and applied magnetic field dependence of magnetization of the FeAl alloys [9, 10], magnetic hysteresis properties (saturation magnetization and coercivity) of the FeAl multilayer samples [11] and magnetic properties of $A 2, B 2, B 32, D 03$ of FeAl alloys [12-22].

In this paper, we consider the unit cell of $B 2-\mathrm{FeAl}$ reported by Plazaola et al. [23] and Yan et al. [24] for the modeling of the B2-FeAl-np. We focus, however, on the experimental $M-T$ results of $B 2-\mathrm{FeAl}$ obtained with a different $\mathrm{Al}$ content $(27.5 \%, 30 \%$, $32.5 \%$ and $35 \%$ ) by Plazaola et al. [23]. They reported that the magnetization and the Curie temperature of $B 2-\mathrm{FeAl}$ decrease with the increase of the $\mathrm{Al}$ content. On the other hand, in the transverse Ising model (TIM), a transverse field $(\Omega)$ causes a similar decrease in the magnetization and the Curie temperature of the magnetic systems. Therefore, for the modeling and investigation of the effects of the $\mathrm{Al}$ content on magnetic properties of $B 2-\mathrm{FeAl}$, we use the TIM within the effective field theory developed by Kaneyoshi [25-27].

\section{Theoretical method}

We use the TIM within the effective field theory developed by Kaneyoshi [25] and focus on the unit cell of $B 2-\mathrm{FeAl}$ reported by Plazaola et al. [23] and Yan et al. [24] for the modeling of the B2-FeAl nanoparticle (B2-FeAl-np), as shown in Fig. 1. We assume that the iron and aluminum atoms of $B 2-F e A l-n p$ are the spin-1/2 Ising particles. According to the nearest neighbors, B2-FeAl-np has five different magnetic atoms and they are $m_{\mathrm{Fe}(1)}$, $m_{\mathrm{Fe}(2)}, m_{\mathrm{Fe}(3)}, m_{\mathrm{Fe}(4)}$ and $m_{\mathrm{Al}}$. Note that the iron atoms have four different magnetizations according to their locations on the $B 2$ unit cell of B2-FeAl-np in Fig. 1. 
According to the Kaneyoshi theory (EFT) [25-27], the Hamiltonian of B2-FeAl-np is given as

$$
\begin{aligned}
\mathcal{H} & =-\sum_{k=1}^{3} J_{k} \sum_{\langle\mathrm{Fe}(k), \mathrm{Fe}(k+1)\rangle} S_{\mathrm{Fe}(k)}^{z} S_{\mathrm{Fe}(k+1)}^{z} \sum_{\langle\mathrm{Al}, \mathrm{Al}\rangle} S_{\mathrm{Al}}^{z} S_{\mathrm{Al}}^{z}-J_{\mathrm{int}} \sum_{k=1}^{4} \sum_{\langle\mathrm{Fe}(k), \mathrm{Al}\rangle} S_{\mathrm{Fe}(k)}^{z} S_{\mathrm{Al}}^{z} \\
& -\left.J_{4}\left(\sum_{k=1}^{4} \sum_{\mathrm{Fe}(k)} S_{\mathrm{Fe}(k)}^{r}+\sum_{\mathrm{Al}} S_{\mathrm{Al}}^{r}\right)\right|_{(r=z) \text { or }(r=x)} \\
& -H\left(\sum_{k}^{4} \sum_{\mathrm{Fe}(k)} S_{\mathrm{Fe}(k)}^{z}+\sum_{\mathrm{Al}} S_{\mathrm{Al}}^{z}\right)
\end{aligned}
$$

where $J_{1}, J_{2}, J_{3}$ and $J_{4}$ are the exchange interaction coefficients between two nearest neighbors, $\mathrm{Fe} 1-\mathrm{Fe} 2, \mathrm{Fe} 2-\mathrm{Fe} 3, \mathrm{Fe} 3-\mathrm{Fe} 4$ and $\mathrm{Al}-\mathrm{Al}$, respectively. The exchange interaction between two nearestneighbor $\mathrm{Fe}$ and $\mathrm{Al}$ atoms is denoted by $J_{\text {int }}$. The values of $J_{i}$, for $i=\{1,2,3,4$, int $\}$, are given by the expression $J_{i}=$ const $/ d_{i}$, where $d_{i}=d_{\text {real }} / 1 \AA$

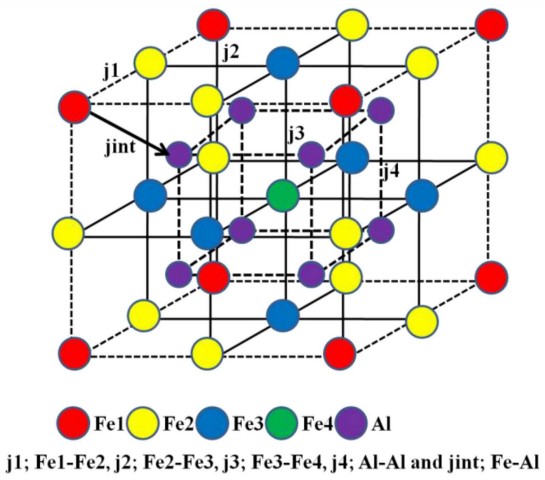

Fig. 1. Schematic representation of the B2-FeAlnp $[23,24]$.

is the reduced distance between two magnetic atoms. For example, $J_{1}=$ const $/ d_{1}=1 / 2.908$ and so on. Next, the Pauli spin operator $S^{r}= \pm 1$ is chosen with respect to the $r$ direction, namely $r=z$ or $r=x$, and $H$ denotes the external magnetic field. In the Kaneyoshi theory [25-27], the magnetizations of $B 2-\mathrm{FeAl}-\mathrm{np}$ are given by

$$
\begin{aligned}
& m_{\mathrm{Fe}(1)}=\left.\left(\cosh \left(J_{1} \nabla\right)+m_{\mathrm{Fe}(2)} \sinh \left(J_{1} \nabla\right)\right)^{3}\left(\cosh \left(J_{\mathrm{int}} \nabla\right)+m_{\mathrm{Al}} \sinh \left(J_{\mathrm{int}} \nabla\right)\right)^{1} F_{\mathrm{S}-\frac{1}{2}}(x)\right|_{x=0}, \\
& m_{\mathrm{Fe}(2)}=\left(\cosh \left(J_{1} \nabla\right)+m_{\mathrm{Fe}(1)} \sinh \left(J_{1} \nabla\right)\right)^{2}\left(\cosh \left(J_{2} \nabla\right)+m_{\mathrm{Fe}(3)} \sinh \left(J_{2} \nabla\right)\right)^{2} \\
& \quad \times\left.\left(\cosh \left(J_{\mathrm{int}} \nabla\right)+m_{\mathrm{Al}} \sinh \left(J_{\mathrm{int}} \nabla\right)\right)^{2} F_{\mathrm{S}-\frac{1}{2}}(x)\right|_{x=0}, \\
& m_{\mathrm{Fe}(3)}=\left(\cosh \left(J_{2} \nabla\right)+m_{\mathrm{Fe}(2)} \sinh \left(J_{2} \nabla\right)\right)^{4}\left(\cosh \left(J_{3} \nabla\right)+m_{\mathrm{Fe}(4)} \sinh \left(J_{3} \nabla\right)\right]^{1} \\
& \quad \times\left.\left(\cosh \left(J_{\mathrm{int}} \nabla\right)+m_{\mathrm{Al}} \sinh \left(J_{\mathrm{int}} \nabla\right)\right)^{4} F_{\mathrm{S}-\frac{1}{2}}(x)\right|_{x=0}, \\
& m_{\mathrm{Fe}(4)}=\left.\left(\cosh \left(J_{3} \nabla\right)+m_{\mathrm{Fe}(3)} \sinh \left(J_{3} \nabla\right)\right)^{6}\left(\cosh \left(J_{\mathrm{int}} \nabla\right)+m_{\mathrm{Al}} \sinh \left(J_{\mathrm{int}} \nabla\right)\right)^{8} F_{\mathrm{S}-\frac{1}{2}}(x)\right|_{x=0}, \\
& m_{\mathrm{Al}}=\left(\cosh \left(J_{4} \nabla\right)+m_{\mathrm{Al}} \sinh \left(J_{4} \nabla\right)\right)^{3}\left(\cosh \left(J_{\mathrm{int}} \nabla\right)+m_{\mathrm{Fe}(1)} \sinh \left(J_{\mathrm{int}} \nabla\right)\right)^{1} \\
& \quad \times\left(\cosh \left(J_{\mathrm{int}} \nabla\right)+m_{\mathrm{Fe}(2)} \sinh \left(J_{\mathrm{int}} \nabla\right)\right)^{3}\left(\cosh \left(J_{\mathrm{int}} \nabla\right)+m_{\mathrm{Fe}(3)} \sinh \left(J_{\mathrm{int}} \nabla\right)\right)^{3} \\
& \quad \times\left.\left(\cosh \left(J_{\mathrm{int}} \nabla\right)+m_{\mathrm{Fe}(4)} \sinh \left(J_{\mathrm{int}} \nabla\right)\right)^{1} F_{\mathrm{S}-\frac{1}{2}}(x)\right|_{x=0},
\end{aligned}
$$

where $\nabla=\frac{\partial}{\partial x}$ is the differential operator. The function $F_{\mathrm{S}-1 / 2}(x)$ for the spin-1/2 Ising particles is defined as:

$F_{\mathrm{S}-\frac{1}{2}}(x)=\frac{(x+H) \tanh \left(\beta \sqrt{(x+H)^{2}+\Omega^{2}}\right)}{\sqrt{(x+H)^{2}+\Omega^{2}}}$,

where $\Omega$ is the transverse field and $\beta=1 /\left(k_{\mathrm{B}} T_{A}\right)$, $k_{\mathrm{B}}$ is the Boltzmann constant and $T_{A}$ denotes the absolute temperature. The units used in the calculation are the reduced temperature $k_{\mathrm{B}} T_{A}$ in $\mathrm{J}$, the reduced transverse field $\Omega$ in $\mathrm{J}$ and the reduced applied field $H$ in $\mathrm{h} / \mathrm{J}$. The total magnetizations of the $\mathrm{Fe}$ atoms, $\mathrm{Al}$ atoms and $\mathrm{B} 2-\mathrm{FeAl}-\mathrm{np}$ are expressed accordingly

$$
\begin{aligned}
& M_{T}^{\mathrm{Fe}}=\frac{1}{27}\left(8 m_{\mathrm{Fe}(1)}+12 m_{\mathrm{Fe}(2)}\right. \\
& \left.\quad+6 m_{\mathrm{Fe}(3)}+m_{\mathrm{Fe}(4)}\right) \\
& M_{T}^{\mathrm{Al}}=\frac{1}{8}\left(8 m_{\mathrm{Al}}\right) \\
& M_{T}^{\mathrm{FeAl}}=\frac{1}{35}\left(8 m_{\mathrm{Fe}(1)}+12 m_{\mathrm{Fe}(2)}+6 m_{\mathrm{Fe}(3)}\right. \\
& \left.\quad+m_{\mathrm{Fe}(4)}+8 m_{\mathrm{Al}}\right) .
\end{aligned}
$$




\section{Theoretical results and discussion}

We have first investigated the effects of the transverse field $\Omega$ on the magnetizations $M_{T}$ and the Curie temperature $T_{\mathrm{C}}$ for the ferromagnetic case (all $J>0)$. The results obtained for the total iron atoms, total aluminium atoms and total $B 2-\mathrm{FeAl}$ nanoparticle at $H=0$ (black solid line) and $H=0.25$ (red solid line) are presented in Fig. 2 . For the lower value of the transverse field $\Omega=0.001$ (see Fig. 2a), the magnetizations and the Curie temperature at $H=0$ are $M_{T}^{\mathrm{Fe}}=M_{T}^{\mathrm{Al}}=M_{T}^{\mathrm{FeAl}}=1$ and $T_{\mathrm{C}}^{\mathrm{Fe}}=T_{\mathrm{C}}^{\mathrm{Al}}=T_{\mathrm{C}}^{\mathrm{FeAl}}=2.95$. Importantly, the total iron atoms $\left(M_{T}^{\mathrm{Fe}}\right)$, total aluminium atoms $\left(M_{T}^{\mathrm{Al}}\right)$ and total B2-FeAl nanoparticle (B2-FeAl-np) have a phase transition at $T_{\mathrm{C}}$ from a ferromagnetic to a paramagnetic state. When the value of $\Omega$ increases, $M_{T}$ and $T_{\mathrm{C}}$ decrease. As a result, we obtain the following: $M_{T}^{\mathrm{Fe}}=0.86453$ for the total iron atoms, $M_{T}^{\mathrm{Al}}=0.96608$ for the total aluminium atoms, $M_{T}^{\mathrm{FeAl}}=0.887742$ for the total B2-FeAl-np and $T_{\mathrm{C}}^{\mathrm{Fe}}=T_{\mathrm{C}}^{\mathrm{Al}}=T_{\mathrm{C}}^{\mathrm{FeAl}}=2.82$ for $\Omega=1$ (see Fig. 2b); $M_{T}^{\mathrm{Fe}}=0.86453$, $M_{T}^{\mathrm{Al}}=0.96608, M_{T}^{\mathrm{FeAl}}=0.887742$ and $T_{\mathrm{C}}^{\mathrm{Fe}}=T_{\mathrm{C}}^{\mathrm{Al}}=T_{\mathrm{C}}^{\mathrm{FeAl}}=2.82$ for $\Omega=2$ (see Fig. $2 \mathrm{c}) ; \quad M_{T}^{\mathrm{Fe}}=M_{T}^{\mathrm{Al}}=M_{T}^{\mathrm{FeAl}}=0.00$ and $T_{\mathrm{C}}^{\mathrm{Fe}}=T_{\mathrm{C}}^{\mathrm{Al}}=T_{\mathrm{C}}^{\mathrm{FeAl}}=0.00$ for $\Omega=3$ (see Fig. $2 \mathrm{~d}$ ). On the other hand, if an external magnetic field is applied ( $H=0.25$, red lines), a paramagnetic state $(M=0)$ disappears and it transforms to the ferromagnetic state $(M \neq 0)$ at $T>T_{\mathrm{C}}$. However, the external magnetic field causes that the magnetization of the ferromagnetic state at $T<T_{\mathrm{C}}$ increases, as seen in Fig. 2a-c. One can observe in Fig. 2d that $B 2-\mathrm{FeAl}-\mathrm{np}$ and its components (Fe and $\mathrm{Al}$ ) are paramagnetic $\left(M_{T}^{\mathrm{Fe}}=M_{T}^{\mathrm{Al}}=M_{T}^{\mathrm{FeAl}}=0.00\right)$ for a high value of the transverse field $\Omega=3$ at $H=0$ (black solid lines). In the case when $H=0.25$, this paramagnetic state transforms to the wholly ferromagnetic state $M_{T}^{\mathrm{Fe}}=0.350266$, $M_{T}^{\mathrm{Al}}=0.52437, M_{T}^{\mathrm{FeAl}}=0.390061$. These transformations clearly show the spin splitting in the ferromagnetic $\mathrm{Fe}$ and $\mathrm{Al}$ atoms in the non-zero external magnetic field $(H=0.25)$.

The effects of the transverse field $\Omega$ on the magnetizations $M_{T}$ and the Curie temperature $T_{\mathrm{C}}$ are shown in Fig. 3 for the total iron atoms, total aluminium atoms and total $B 2-\mathrm{FeAl}$-np. The results refer to the antiferromagnetic case (i.e., $J_{\text {int }}<0$ ), i.e., antiferromagnetism between the Fe (shell) and Al (core) atoms at $H=0$ (black solid line) and $H=0.25$ (red solid line). In fact, all magnetization curves of the total iron atoms $M_{T}^{\mathrm{Fe}}$, total aluminium atoms $M_{T}^{\mathrm{Al}}$ and total $B 2-\mathrm{FeAl}-\mathrm{np} M_{T}^{\mathrm{FeAl}}$ differ much more from each other than in the ferromagnetic case (see Fig. 2). At a lower value of $\Omega=0.001$, as presented in Fig. 3a, the magnetization values are: $M_{T}^{\mathrm{Fe}}=1$ for the total iron atoms, $M_{T}^{\mathrm{Al}}=-1$ for the total aluminium atoms and $M_{T}^{\mathrm{FeAl}}=0.542857$ for the total B2-FeAl-np. When the value of $\Omega$ increases, the magnetization

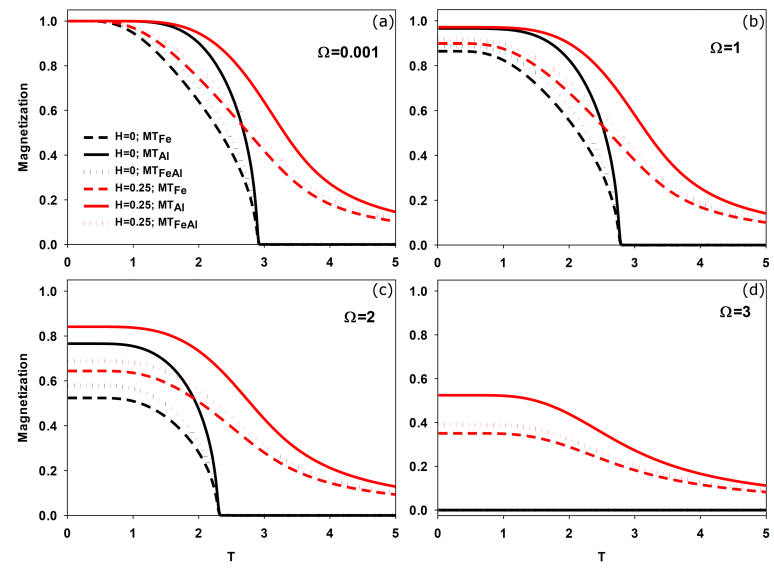

Fig. 2. $M-T$ curves of the $B 2-F e A l-n p$ in the ferromagnetic case for $\Omega=0.001,1,2$ and 3 at $H=0$ (black lines) and $H=0.25$ (red lines).

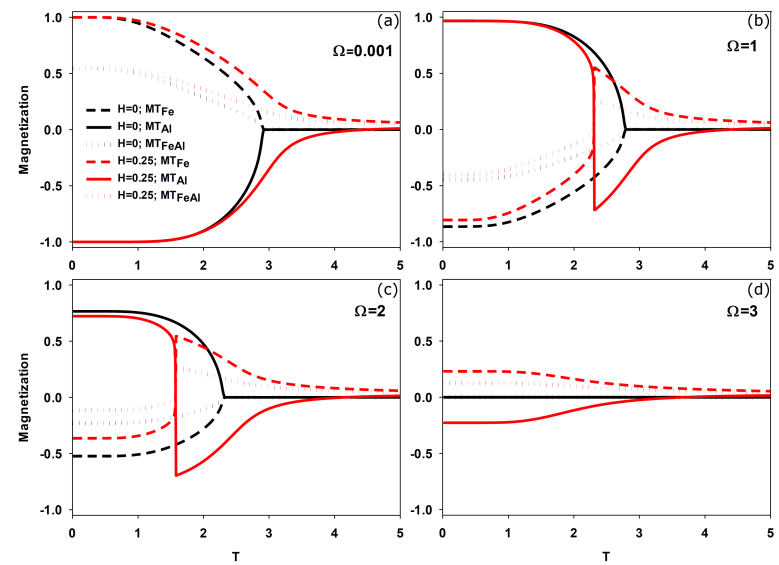

Fig. 3. $\quad M-T$ curves of the $B 2-F e A l-n p$ in the antiferromagnetic case for $\Omega=0.001,1,2$ and 3 at $H=0$ (black lines) and $H=0.25$ (red lines).

values become as follows: $M_{T}^{\mathrm{Fe}}=-0.86453$, $M_{T}^{\mathrm{Al}}=0.96608, M_{T}^{\mathrm{FeAl}}=-0.44611$ for $\Omega=1$ (see Fig. 3b); $M_{T}^{\mathrm{Fe}}=-0.523692, M_{T}^{\mathrm{Al}}=0.765827$, $M_{T}^{\mathrm{FeAl}}=-0.228945$ for $\Omega=2$ (see Fig. 3c); and $M_{T}^{\mathrm{Fe}}=0.00, \quad M_{T}^{\mathrm{Al}}=0.00, \quad M_{T}^{\mathrm{FeAl}}=0.00$ for $\Omega=3$ (see Fig. 3d).

It is worth to note that the $M_{T}$ value of the iron atoms as well as of $B 2-\mathrm{FeAl}-\mathrm{np}$ for the lower value of the transverse field $(\Omega=0.001)$ is positive whereas the magnetization of the aluminium atoms is negative (see Fig. 3a). The situation, however, is inverse at the higher values of the transverse field ( $\Omega=1,2$ and 3 , see Fig. $3 \mathrm{~b}-\mathrm{d}$, respectively) and the external magnetic field $H=0$. Further, if one applies the external magnetic field $H=0.25$ (red lines), then the paramagnetic state $(M=0)$ disappears and it transforms to the antiferromagnetic state $(M \neq 0)$ at $T>T_{\mathrm{C}}$. Also note that the B2-FeAl-np (see Fig. 3b) and its counter-components (Fe and $\mathrm{Al}$, see Fig. 3c) experience a first-order phase transition at $T_{f}=2.31$ 


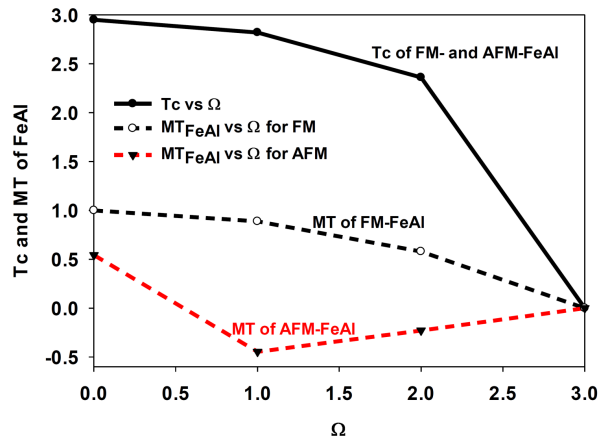

Fig. 4. $T_{\mathrm{C}^{-}} \Omega$ and $M_{T^{-}} \Omega$ curves of the FM and AFM B2-FeAl-np.

and 1.58 , respectively. The magnetization $M_{T}$ corresponds to the iron atoms and $B 2-\mathrm{FeAl}-\mathrm{np}$ transforms from positive to negative, whereas $M_{T}$ of the aluminium atoms transforms from a negative to a positive value for $\Omega=1$ and 2 at $H=0.25$. In Fig. 3d, however, one can see that the paramagnetic state transforms completely to the antiferromagnetic state for $\Omega=3\left(M_{T}^{\mathrm{Fe}}=0.22759\right.$, $M_{T}^{\mathrm{Al}}=-0.221962, M_{T}^{\mathrm{FeAl}}=0.124835$. These transformations clearly show that the spin splitting of the antiferromagnetic $\mathrm{Fe}$ and $\mathrm{Al}$ atoms is entirely different from this of the ferromagnetic case (see Fig. 2d) for the non-zero external magnetic field $H=0.25$.

Figure 4 shows the transverse field dependence of the magnetizations and the Curie temperature of the total B2-FeAl-np for the ferromagnetic and antiferromagnetic case. As it can be observed, the magnetization and the Curie temperature decrease as the value of $\Omega$ increases. Let us focus now on this point. The experimental $M-T$ results of B2-FeAl-np [23] were obtained for different $\mathrm{Al}$ contents $(27.5 \%, 30 \%, 32.5 \%$ and $35 \%)$. It was found that the magnetization and the Curie temperature of $B 2-\mathrm{FeAl}-\mathrm{np}$ decrease as the $\mathrm{Al}$ content increases. The qualitative agreement between the experimental and theoretical results indicates that the increase of the $\mathrm{Al}$ content in $B 2-\mathrm{FeAl}-\mathrm{np}$ is responsible for the increase in the transverse field in $B 2-F e A l-n p$. It also causes a decrease of both the magnetization (dashed black line) and the Curie temperature (solid black line) of B2-FeAl-np.

\section{Conclusions}

We have investigated the effects of the transverse field on the magnetizations and the Curie temperature of the $B 2$ nanostructure of the B2-FeAl-np by using the effective field theory (the Kaneyoshi theory) at a zero and non-zero external magnetic field. The main conclusions are:

1. The magnetizations and the Curie temperature decrease as the transverse field increases.

2. Our theoretical $M-T$ results of $B 2-\mathrm{FeAl}-\mathrm{np}$ agree qualitatively with the experimental
$M-T$ results of the off-stoichiometric $\mathrm{FeAl}$ with $B 2$ structure for a different $\mathrm{Al}$ content $(27.5 \%, 30 \%, 32.5 \%$ and $35 \%)$ by Plazaola et al. [23].

3. The qualitative agreement between the theoretical and experimental results of the magnetizations and the Curie temperature of $B 2$ FeAl and B2-FeAl-np might suggest that an increase of the $\mathrm{Al}$ content in $B 2-\mathrm{FeAl}$ causes an increase in the transverse field of $B 2-\mathrm{FeAl}$.

4. For the antiferromagnetic case, different negative magnetization behaviors are obtained in $B 2-\mathrm{FeAl}$-np and its components (Fe and $\mathrm{Al}$ ). These differences result from the transverse field and external magnetic field.

5. B2-FeAl-np and its components (Fe and $\mathrm{Al}$ ) undergo a first-order phase transition at $T_{f}=2.31$ and 1.58 for $\Omega=1$ and 2 in a nonzero $(H=0.25)$ external magnetic field.

6 . The spin splitting of the paramagnetic Fe and $\mathrm{Al}$ atoms is very different in the ferromagnetic and antiferromagnetic cases for $\Omega=3$ in a non-zero $(H=0.25)$ external magnetic field at $T>T_{\mathrm{C}}$.

\section{Acknowledgments}

This study was supported by the Scientific Research Projects Unit of Dumlupinar University under grant No. 2019/28. We would also like to express our gratitude to the staff of the Computer Center of Erciyes University.

\section{References}

[1] Y. Murakami, K. Niitsu, T. Tanigaki, R. Kainuma, H.S. Park, D. Shindo, Nature Commun. 5, 4133 (2014).

[2] D. Wen, S. Xiao-Xiang, T. Shao-Xi, L. Yu-Xia, X. Ding-Kang, H. Yu-Yang, Chin. Phys. C 37, 128201 (2013).

[3] M. Friak, J. Neugebauer, Intermetallics 18, 1316 (2010).

[4] Y.-Y. Huang, L.-Y. Qin, Q.-G. Tian, Y.-X. Li, W. Deng, Adv. Mater. Res. 399, 908 (2012).

[5] S.N. Kaul, A.C. Abhyankar, D.V. Sridhar Rao, K. Muraleedharan, A.K. Nigam, L.F. Barquín, Mater. Sci. Forum 635, 111 (2010).

[6] R. Katoh, K. Nonaka, K. Sumiyama, D.-L. Peng, T. Hihara, Mater. Trans. 49, 1830 (2008).

[7] E.V. Voronina, E.P. Elsukov, A.V. Korolev, A.V. Zagainov, A.E. Elsukova, Phys. Met. Metall. 104, 351 (2007).

[8] E.P. Elsukov, E.V. Voronina, A.V. Korolev, A.E. Elsukova, S.K. Godovikov, Phys. Met. Metall. 104, 35 (2007). 
[9] E.V. Voronina, E.P. Elsukov, S.K. Godovikov, A.V. Korolev, A.E. Elsukova, Phys. Met. Metall. 109, 417 (2010).

[10] Q. Zeng, I. Baker, Intermetallics 14, 396 (2006).

[11] R. Brajpuriya, S. Tripathi, S.M. Chaudhari, Solid State Commun. 134, 479 (2005).

[12] D. Wu, I. Baker, P.R. Munroe, Intermetallics 12, 851 (2004).

[13] E. Apiñaniz, F. Plazaola, J.S. Garitaonandia, Europ. Phys. J. B 31, 167 (2003).

[14] J.Y.Y. Lin, B. Fultz, Philos. Mag. 83, 2621 (2003).

[15] D. Wu, P.R. Munroe, I. Baker, Philos. Mag. 83, 295 (2003).

[16] G.P. Das, B.K. Rao, P. Jena, S.C. Deevi, Phys. Rev. B 66, 184203 (2002).

[17] M. Kass, C.R. Brooks, D. Falcon, D. Basak, Intermetallics 10, 951 (2002).

[18] J. Deniszczyk, Acta Phys. Pol. A 98, 543 (2000).
[19] M. Fujii, K. Saito, K. Wakayama, M. Kawasaki, T. Yoshioka, T. Isshiki, K. Nishio, M. Shiojiri, Philos. Mag. A 79, 2013 (1999).

[20] B. Sprušil, B. Chalupa, Intermetallics 7 , 635 (1999).

[21] S. Takahashi, Y. Umakoshi, J. Phys. Condens. Matter 3, 5805 (1991).

[22] T. Zak, T. Zemčík, Czechoslov. J. Phys. 35, 1362 (1985).

[23] F. Plazaola, E. Apinaniz, D. Martin Rodriguez, E. Legarra, J.S. Garitaonandia, in: Fe-Al Alloys Magnetism, Advanced Magnetic Materials, Ed. L. Malkinski, 2012.

[24] X. Yan, A. Grytsiv, P. Rogl, V. Pomjakushin, M. Palm, J. Phase Equilib. Diff. 29, 500 (2008).

[25] R. Honmura, T. Kaneyoshi, J. Phys. C Solid State Phys. 12, 3979 (1979).

[26] T. Kaneyoshi, Acta Phys. Pol. A 83, 703 (1993).

[27] T. Kaneyoshi, M. Jascur, I.P. Fittipaldi, Phys. Rev. B 48, 250 (1993). 